\title{
Total degradation of a DVB-S2 satellite system with analog transparent optical feeder link
}

\author{
Alexis A. Dowhuszko ${ }^{1}$, Senior Member, IEEE, Alberto Mengali ${ }^{2}$, \\ Pantelis-Daniel Arapoglou ${ }^{2}$, Ana I. Pérez-Neira ${ }^{1,3}$, Senior Member, IEEE \\ ${ }^{1}$ Centre Tecnològic de Telecomunicacions de Catalunya (CTTC/CERCA), Barcelona, Spain \\ ${ }^{2}$ European Space Research and Technology Centre (ESTEC), European Space Agency (ESA), Noordwijk, The Netherlands \\ ${ }^{3}$ Department of Signal Theory and Communications, Universitat Politècnica de Catalunya (UPC), Barcelona, Spain \\ Email: \{alexis.dowhuszko, ana.perez\}@cttc.es; \{alberto.mengali, pantelis-daniel.arapoglou\}@esa.int
}

\begin{abstract}
This paper studies the End-to-End (E2E) performance of a High Throughput Satellite (HTS) system that utilizes a transparent (non-regenerative) optical feeder link to transport the DVB-S2 signals associated with the large number of spotbeams in the radio access link. Two main sources of nonlinearity are considered to characterize the effect that the satellite forward link has in the DVB-S2 signal, namely the optical MachZehnder Modulator (MZM) in the gateway and the High Power Amplifier (HPA) in the satellite. Digital Pre-Distortion (DPD) and linear equalization are also implemented to mitigate the impact of the non-linear distortion and the inter-symbol interference on the Packet Error Rate (PER) of the received DVB-S2 signal. The performance analysis is carried out using the Total Degradation (TD) as performance indicator. As expected, the Input Back-Off (IBO) of the HPA and the modulation index of the MZM should be jointly optimized according to the DVB-S2 MODCOD that is selected for transmission. Therefore, the stronger is the Forward Error Correction (FEC) code that is used, the lower is the IBO and the higher is the modulation index that can be utilized to minimize the impact of non-linearities in the E2E performance.
\end{abstract}

\section{INTRODUCTION}

High Throughput Satellite (HTS) systems are constantly evolving to provide $5 \mathrm{G}$ data services in remote areas where terrestrial mobile access is not possible or economically viable [1]. In order to support an aggregate data rate in the range of few Terabit-per-second (Tbps), the satellite should utilize a very large number of spot-beams with an aggressive frequency reuse factor in the radio access link (i.e., the link between the satellite and the user terminals) [2]. In this situation, the feeder link (i.e., the link between the gateway and the satellite) becomes the bottleneck of the HTS system, as it must support a point-to-point data rate as large as the aggregate data rate of the spot-beams in the radio access part. Different solutions have been proposed to address this problem, such as adding new Radio Frequency (RF) bands for the feeder link, deploying multiple satellite gateways on different geographical locations, and using optical wireless technology in the feeder link [3]. This paper focuses on the latter option, known as Optical Feeder Link, as the point-to-point data rate that can be supported with optical wireless technology is few orders of magnitude larger than with RF. In addition, optical wireless systems do not need license for operation and simplifies the

This work has been funded by the Ministry of Science, Innovation, and Universities of Spain under Project TERESA-TEC2017-90093-C3-1-R (AEI/FEDER, UE), by the Catalan government under grant 2017 SGR 1479, and by the European Space Agency (ESA) under the SATNEX IV framework. The views expressed herein cannot be taken to reflect ESA's official opinion. control of optical interference of co-located wireless links due to the high directivity gain that optical transceivers have [4].

The design of the optical feeder link depends on the approach that is used to forward the information [5]. In the fully regenerative payload case, the optical feeder link terminates in the satellite; therefore, robust Forward Error Correction (FEC) coding can be used to correct the long error bursts that the turbulent optical wireless channel introduces [6]. Moreover, this approach enables the implementation of advanced signal processing mechanisms (e.g., beam precoding) onboard the satellite, making possible to achieve even higher spectral/energy efficiencies in the HTS system [7]. In return, the fully regenerative payload demands the highest processing power on-board the satellite and provides the least flexibility to adapt to the modifications that the DVB-S2 standard could experience during the satellite life span. Therefore, transparent non-regenerative bent-pipe solutions should be initially favored, regardless they are analog or digital transparent [5].

In the analog transparent payload, also known as Radioover-Free Space Optics (FSO) solution, the instantaneous value of the radio signal modulates the intensity of the Laser Diode (LD) [8]. In the digital transparent payload, on the other hand, the I/Q components of the radio baseband signal are oversampled and quantized to guarantee an adequate Signalto-Distortion power Ratio (SDR). After that, the resulting sequence of information bits is used to modulate digitally the optical carrier of the feeder link [5]. In the digital transparent payload, only limited signal processing capabilities are required on-board the satellite to reconstruct the complexvalued I/Q baseband signal; moreover, FEC encoding can be used to protect the digitalized samples of the baseband signal against the turbulence-induced fading of the optical wireless channel. Unfortunately, the digital transparent approach limits the aggregate data rate that the HTS system can support, as the digitalization process expands many times the original bandwidth of the complex I/Q baseband signal [9].

So far, HTS systems have been designed to implement an over-dimensioned feeder link (on RF bands), such that the End-to-End (E2E) data rate was hard-limited by the channel gain in the radio access part. However, as the aggregate data rate of the HTS system grows, and optical technologies are needed to implement the feeder link, the estimation of the E2E data rate should also take into account the non-linear distortion and noise that are added in the optical feeder link. That is, the hybrid optical-radio wireless link that results when 


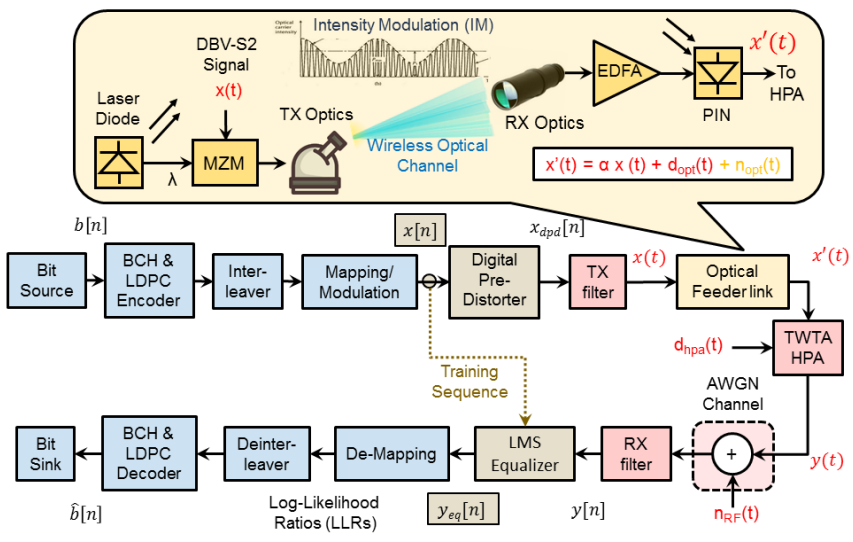

Fig. 1: Bock diagram of a HTS system with optical feeder link. Blue and red blocks identify the digital and analog processing, respectively. Orange blocks represent the optical feeder link, whereas the brown blocks perform pre- and post-distortion compensation.

the optical feeder link is concatenated with the RF access link should be jointly optimized. For example, pre- and postdistortion compensation [10] could be applied to the DVB-S2 radio signal that is transmitted by the satellite gateway and is received by the user terminal, respectively, such that the non-linear distortion and noise that the optical components (i.e., Mach-Zehnder Modulator, Photodetector, etc.) and the electrical components (i.e., High Power Amplifier, Low Noise Amplifier, etc.) introduce are jointly compensated.

The rest of the paper is organized as follows: Section II introduces the system model and describes the most important blocks of the DVB-S2 communication standard, the analog transparent optical feeder link, and the pre- and post-distortion compensation techniques. Section III describes the theoretical framework for the performance evaluation, with emphasis on the definition the Total Degradation (TD) as indicator and the modeling of the in-band distortion that the optical feeder link introduces in the received DVB-S2 signal. Simulation results, including their corresponding analysis, are presented in Section IV. Finally, conclusions are drawn in Section V.

\section{System ModeL}

The simplified system model of the HTS system is illustrated in Fig. 1. It consists of a DVB-S2 satellite communication system [11], where the blue and red boxes represent the digital and analog processing blocks, respectively. The elements of the optical feeder link (orange boxes) implement an analog transparent (non-regenerative) transportation of the baseband DVB-S2 signal, which is up-converted to an Intermediate Frequency (IF) before its transmission. The brown boxes provide digital (data) pre-distortion and postdistortion (equalization) compensation, to mitigate the effects that the Mach-Zehnder Modulator (MZM) and High Power Amplifier (HPA) introduce. For the sake of simplicity, ideal carrier and timing synchronizations are assumed.

\section{A. Digital signal processing (DVB-S2 standard)}

The digital signal processing blocks, which are represented with blue boxes in Fig. 1, focus on the encoding, interleaving, and modulation of the information bits to be transmitted.
1) Forward Error Correction: The bits of the source are grouped into packets, whose size depends on the Modulation and Coding (MODCOD) that is selected to transmit the DVB-S2 frame. After that, each packet undergoes FEC encoding, which concatenates an outer Bose-ChaudhuriHocquenghem $(\mathrm{BCH})$ code with an inner Low-Density Parity Check (LDPC) code. The DVB-S2 standard defines eleven FEC code rates, but only a sub-set of them is supported by each modulation scheme [11].

2) Modulation and bit-to-symbol mapping: The DVB-S2 standard defines four modulation schemes, namely: QPSK, 8-PSK, 16-APSK, and 32-APSK [11]. When compared to $M$-QAM, $M$-APSK has a lower Peak-to-Average Power Ratio (PAPR) and, due to that, is more robust against the effects of the non-linear distortion that is introduced. More precisely, 16-APSK is composed by an inner 4-PSK ring surrounded by an outer 12-PSK ring, wheras 32-APSK is composed by an inner 4-PSK ring, an intermediate 12-PSK ring, and an outer 16-PSK ring. The separation between rings is specified in the DVB-S2 standard [11], and has been selected to minimize the Packet Error Rate (PER) of the satellite system.

In order to map the incoming bits with the constellation points of the modulation scheme, an interleaving process is applied in 8-PSK, 16-APSK, and 32-APSK and, after that, the interleaved bits are mapped into symbols of a constellation with Grey mapping [11]. In this paper, we focus on the higher order modulation schemes (i.e., 16- and 32-APSK), as the nonlinear distortion power impacts more notably the resulting PER due to the constellation points are the most closely packed.

3) Iterative de-mapping: The DVB-S2 standard defines an iterative processing for the detection of the sequence of received bits. The complex received signal samples $\mathbf{r}=(x, y)$ are de-mapped by a Log-Likelihood Ratio (LLR) metric that is computed for each of the $K$ bits of every $M$-ary constellation point. More precisely, the LLR for data bit $b_{i}$ given received signal $\mathbf{r}$ is defined as the logarithm of the ratio of the probability for the bit taking its two possible values, i.e.,

$$
\begin{aligned}
L\left(b_{i} \mid \mathbf{r}\right) & :=\log _{\mathrm{e}}\left[\frac{P\left(b_{i}=1 \mid \mathbf{r}\right)}{P\left(b_{i}=0 \mid \mathbf{r}\right)}\right] \\
& =\log _{\mathrm{e}}\left[\sum_{s \in \mathcal{S}_{1}} \exp \left(-\frac{\left(x-s_{\mathrm{x}}\right)^{2}+\left(y-s_{\mathrm{y}}\right)^{2}}{\sigma_{\mathrm{n}}^{2}}\right)\right] \\
& -\log _{\mathrm{e}}\left[\sum_{s \in \mathcal{S}_{0}} \exp \left(-\frac{\left(x-s_{\mathrm{x}}\right)^{2}+\left(y-s_{\mathrm{y}}\right)^{2}}{\sigma_{\mathrm{n}}^{2}}\right)\right],
\end{aligned}
$$

where $s_{\mathrm{x}}\left(s_{\mathrm{y}}\right)$ is the value of the in-phase (quadrature) component of the ideal transmitted symbol, $\mathcal{S}_{0}\left(\mathcal{S}_{1}\right)$ is the set of ideal symbols with bit ' 0 ' (' 1 ') at the given position, and $\sigma_{\mathrm{n}}^{2}$ is the variance of the complex noise in the received baseband signal. Intuitively, the sign of the LLR indicates if the bit is more likely to be ' 1 ' or ' 0 ', whereas the magnitude indicates the likelihood that the sign of the LLR is the correct value.

\section{B. Analog signal processing (DVB-S2 standard)}

The analog signal processing blocks, which are represented with red boxes in Fig. 1, focus on the generation of the continuous time pulses in transmission, the analog amplification on-board the satellite, and the matched-filtering in reception. 
1) Transmit and receive filters: The DVB-S2 standard defines a conventional Square-Root Raised-Cosine (SRRC) function for pulse-shaping in transmission and matched-filtering in reception. Three different roll-off factors can be selected:

- $\alpha=0.35$ (inherited from the former DVB-S standard),

- $\alpha=0.25$ and 0.20 (added in the DVB-S2 standard).

As expected, the lower is the roll-off factor, the longer is the pulse duration in the time domain and the more concentrated is the spectral pulse energy in the main frequency lobe.

2) High Power Amplifier: The HPA non-linear response is given by its $\mathrm{AM} / \mathrm{AM}$ and $\mathrm{AM} / \mathrm{PM}$ conversion characteristics, which represent the way in which the input signal amplitude affects the amplitude and phase of the output signal, respectively. Let us assume that $x(t)=\rho(t) \exp [\mathrm{j} \phi(t)]$ is the complex input. Then, the signal at the HPA output becomes

$$
y(t)=G[x(t)]=F[\rho(t)] \exp \{\mathrm{j} \phi(t)+\mathrm{j} \theta[\rho(t)]\},
$$

where $F[\cdot]$ and $\theta[\cdot]$ are the AM/AM and AM/PM conversion characteristics, which are defined in the DVB-S2 standard [11].

\section{Analog transparent optical feeder link (non-regenerative)}

The optical feeder link is represented with orange boxes in Fig. 1, and implements an analog (non-regenerative) transportation of the DVB-S2 signal over an IM/DD FSO link.

1) Intensity Modulation (IM) of the optical carrier: The transportation of the complex DVB-S2 baseband (BB) signal over the optical feeder link is achieved by modulating the instantaneous intensity of the light beam that the LD emits at wavelength $\lambda$. To achieve this goal, the complex DVB-S2 signal must be first converted into a passband (PB) signal, using for this purpose an up-conversion stage. We now present the mathematical formulas that explain this process in detail.

Let the block of symbols that the DVB-S2 carrier transmits during the time frame with index $i$ be given by

$$
x_{\mathrm{bb}, i}^{\mathrm{dvb}-\mathrm{s} 2}(t)=\sum_{k} x_{i}^{\mathrm{dvb}-\mathrm{s} 2}[k] g_{\mathrm{tx}}\left(t-k T_{\mathrm{s}}\right),
$$

where $k$ is the position of the data symbol in the DVB-S2 frame, $T_{\mathrm{s}}$ is the symbol time, and $g_{\mathrm{tx}}(t)$ is the SRRC transmit pulse-shaping filter with roll-off factor $\alpha$. After that, the complex baseband signal is up-converted to the IF $f_{\text {if }}$ to obtain

$$
\begin{aligned}
s_{\mathrm{pb}, i}^{\mathrm{dvb}-\mathrm{s} 2}(t) & =\operatorname{Re}\left\{x_{\mathrm{bb}, i}^{\mathrm{dvb}-\mathrm{s} 2}(t) \exp \left(\mathrm{j} 2 \pi f_{\mathrm{if}} t\right)\right\} \\
& =a_{\mathrm{bb}, i}^{\mathrm{dvb}-\mathrm{s} 2}(t) \cos \left(\omega_{\mathrm{if}} t+\varphi_{\mathrm{bb}, i}(t)\right),
\end{aligned}
$$

where $\omega_{\text {if }}=2 \pi f_{\text {if }}$ is the angular frequency and

$$
a_{\mathrm{bb}, i}^{\mathrm{dvb}-\mathrm{s} 2}(t)=\left|x_{\mathrm{bb}, i}^{\mathrm{dvb}-\mathrm{s} 2}(t)\right|, \quad \varphi_{\mathrm{bb}, i}(t)=\arg \left\{x_{\mathrm{bb}, i}^{\mathrm{dvb}-\mathrm{s} 2}(t)\right\},
$$

are the envelope and phase on the IF carrier, respectively.

The input voltage that is applied to the control terminals of the external optical MZM is given by

$$
v_{\mathrm{mzm}}=V_{\mathrm{B}}+\beta s_{\mathrm{pb}}(t)\left(V_{\pi} / \pi\right),
$$

where $V_{\mathrm{B}}$ and $V_{\pi}$ are the bias and half-wave voltages of the MZM, respectively, $\beta$ is the intensity modulation index, and $s_{\mathrm{pb}}(t)=\sum_{i} s_{\mathrm{pb}, i}^{\mathrm{dvb}-\mathrm{s} 2}(t) / \sqrt{\mathbb{E}\left\{\sum_{i}\left|s_{\mathrm{pb}, i}^{\mathrm{dvb}-\mathrm{s} 2}(t)\right|^{2}\right\}}$ is the normalized passband DVB-S2 signal. In the latter formula, $\mathbb{E}\{\cdot\}$ represents the mathematical expectation of the corresponding time-domain signal. The specific choice of $\beta$ controls the range over which the MZM works and, at the same time, the received power and the level of non-linear distortion that is observed in the radio signal after its photodetection on-board the satellite.

Let $P_{\mathrm{o}}$ denote the average optical power of the LD that feeds the MZM. Then, based on the MZM transfer function, the envelope of the optical intensity at the MZM output becomes

$$
\begin{aligned}
\left|p_{\mathrm{o}}(t)\right| & =\frac{P_{\mathrm{o}}}{2}\left[1+\cos \left(\frac{\pi}{V_{\pi}} v_{\mathrm{mzm}}(t)\right)\right] \\
& =\frac{P_{\mathrm{o}}}{2}\left[1+\cos \left(\frac{\pi V_{\mathrm{B}}}{V_{\pi}}+\beta s_{\mathrm{pb}}(t)\right)\right] .
\end{aligned}
$$

When an appropriate bias point is used, e.g. $V_{\mathrm{B}}=\left(3 V_{\pi}\right) / 2$, the intensity modulated optical carrier attains the form

$$
\begin{aligned}
p_{\mathrm{o}}(t) & =\operatorname{Re}\left\{\left|p_{\mathrm{o}}(t)\right| \exp \left(\mathrm{j} \omega_{\mathrm{o}} t\right)\right\} \\
& =\left(P_{\mathrm{o}} / 2\right)\left[1+\sin \left(\beta s_{\mathrm{pb}}(t)\right)\right] \cos \left(\omega_{\mathrm{o}} t\right),
\end{aligned}
$$

where $\left(P_{\mathrm{o}} / 2\right) \cos \left(\omega_{\mathrm{o}} t\right)$ is the unmodulated optical carrier with angular frequency $\omega_{\mathrm{o}}$ that is transmitted with the optical sidebands that the DVB-S2 modulating signal generates.

2) Optical wireless channel: It is mainly characterized by Free Space Loss (FSL) and atmospheric turbulence.

The FSL represents the largest power loss and is given by

$$
L_{\mathrm{o}, \mathrm{fsl}}=\lambda^{2} /(4 \pi R)^{2},
$$

where $R$ is the range of the optical wireless link at wavelength $\lambda$ (i.e., $R \approx 39000 \mathrm{~km}$ for a GEO satellite system). Besides the FSL, there are additional losses when the signal propagates through a lossy medium, such as the low layers of the Earth's atmosphere, particularly in case of weather conditions that reduce the visibility. For clear sky, atmospheric absorption and scattering only affects the attenuation of the optical wireless signal significantly at low elevation angles.

The atmospheric turbulence is caused by the mixing of warm and cold air, generating small variations on the refractive index of the signal path that induce fluctuations on the received optical intensity. In case of weak turbulence, the statistics of the received optical intensity can be approximated with a LogNormal distribution. Similarly, the exponential distribution for strong turbulence and the Gamma-Gamma distribution for a wider range of turbulence conditions can also be utilized. Although the impact of these stochastic models have been widely studied in the literature, we tackle this issue by reserving few $\mathrm{dBs}$ of the optical feeder link budget to address the effect of turbulence on the received intensity (i.e., system losses).

3) Direct Detection (DD) of the IM optical signal: The optical signal that illuminates the Photodetector (PD) in the satellite generates an electrical current that is equal to

$$
i_{\mathrm{D}}(t)=\mu \frac{G_{\mathrm{o}, \mathrm{tx}} G_{\mathrm{o}, \mathrm{rx}} G_{\mathrm{o}, \mathrm{edfa}}}{L_{\mathrm{o}, \mathrm{fsl}} L_{\mathrm{o}, \mathrm{sys}}}\left|p_{\mathrm{o}}(t)\right|,
$$

where $\mu[\mathrm{A} / \mathrm{W}]$ is the $\mathrm{PD}$ responsivity, $G_{\mathrm{o}, \text { edfa }}$ is the gain of the Erbium-Doped Fiber Amplifier (EDFA), and $L_{\mathrm{o} \text {,sys }}$ contemplates the system losses in the optical feeder link. The detected current at the PD output can be divided into two terms, i.e., $i_{\mathrm{D}}(t)=I_{\mathrm{D}}+i_{\mathrm{d}}(t)$, where

$$
I_{\mathrm{D}}=\mathbb{E}\left\{i_{\mathrm{D}}(t)\right\}=\mu \frac{G_{\mathrm{o}, \mathrm{tx}} G_{\mathrm{o}, \mathrm{rx}} G_{\mathrm{o}, \mathrm{edfa}}}{L_{\mathrm{o}, \mathrm{fsl}} L_{\mathrm{o}, \mathrm{sys}}} \frac{P_{\mathrm{o}}}{2}
$$


is the $\mathrm{DC}$ component that is constant regardless of $\beta$, and

$$
i_{\mathrm{d}}(t)=i_{\mathrm{D}}(t)-I_{\mathrm{D}}=I_{\mathrm{D}} \sin \left(\beta s_{\mathrm{pb}}(t)\right)
$$

is the $\mathrm{AC}$ component that depends on $\beta$ and on the instantaneous value of the modulating DVB-S2 signal. Finally, the Optical Signal-to-Noise Ratio (OSNR) at the PD input is

$$
\text { OSNR }=\mathbb{E}\left\{\left|i_{\mathrm{d}}(t)\right|^{2}\right\} / \mathbb{E}\left\{\left|n_{\mathrm{o}}(t)\right|^{2}\right\},
$$

where

$$
\begin{aligned}
\mathbb{E}\left\{\left|n_{\mathrm{o}}(t)\right|^{2}\right\} & =\mathbb{E}\left\{\left|i_{\text {shot }}(t)\right|^{2}\right\}+\mathbb{E}\left\{\left|i_{\text {thermal }}(t)\right|^{2}\right\} \\
& +\mathbb{E}\left\{\left|i_{\text {rin }}(t)\right|^{2}\right\}+\mathbb{E}\left\{\left|i_{\text {beat }}(t)\right|^{2}\right\}
\end{aligned}
$$

includes the contribution of all sources of noise added in the optical feeder link, namely shot noise (due to the signal, amplified spontaneous emission noise, background optical noise and dark current noise), thermal noise, Relative Intensity Noise (RIN) of the LD, and beat noise due to the combination of the desired optical signal and the spontaneous emissions [12], [13]. When the received optical power is between -110 and $-90 \mathrm{dBm}$, it can be shown that the beat noise between the useful signal and the Amplified Spontaneous Emission (ASE) noise dominates the OSNR performance, i.e.,

$$
\begin{aligned}
\mathbb{E}\left\{\left|n_{\mathrm{o}}(t)\right|^{2}\right\} & \approx \mathbb{E}\left\{\left|i_{\text {beat }}(t)\right|^{2}\right\}=i_{\mathrm{sig}-\mathrm{sp}}^{2}+i_{\mathrm{sp}-\mathrm{sp}}^{2} \\
& \approx i_{\mathrm{sig}-\mathrm{sp}}^{2}=4 I_{\mathrm{D}} I_{\mathrm{ASE}}\left(B_{\mathrm{o}} / B_{\mathrm{e}}\right),
\end{aligned}
$$

where $B_{\mathrm{O}}\left(B_{\mathrm{e}}\right)$ is the bandwidth of the optical (electrical) signal at the input (output) of the $\mathrm{PD}$, and $I_{\mathrm{ASE}}=\mu G_{\mathrm{o} \text {,edfa }} P_{\mathrm{ASE}}$ is the DC component generated by the ASE noise when $P_{\mathrm{ASE}}$ is the equivalent noise power at the EDFA input [13].

\section{Digital pre- and post-distortion compensation}

The techniques to compensate the distortion, which are represented with brown boxes in Fig. 1, are applied in the digital samples of the data symbols that are transmitted and received to mitigate the impact of the MZM and HPA nonlinear response in the TD of the DVB-S2 system.

1) Pre-distortion techniques: These techniques modify the transmitted signal to mitigate the non-linear distortion that is added during the transmission. This can be done in various ways [10], leading to techniques that seek the inversion of the non-linear channel response by operating on the baseband symbols (i.e. data digital pre-distortion) or baseband signal (i.e., signal analog pre-distortion). In order to keep this study simple, we focus on a simple Digital Pre-Distortion (DPD) scheme that modifies the constellation of symbols before the pulse-shaping filter is applied. This way, the out-of-band interference is avoided, and the DPD technique can be easily implemented with a Lookup Table (LUT), whose elements are optimized to minimize the Mean Square Error (MSE) between the actual distorted received symbol samples and the ideal non-distorted symbol samples that should be received.

2) Equalization techniques: In an actual setting, the DPD block cannot fully compensate the non-linear effects because actual non-linear channel models utilize limited degrees of freedom and, sometimes, the equivalent channel when concatenating the MZM and HPA is not always invertible. Though non-linear equalization techniques could be considered to improve the received signal, in this paper we use a singletap Least Mean Square (LMS) linear equalizer in reception, which is trained assuming that given symbols of the DVB-S2 frame are known a priori in reception to train the equalizer.

\section{Distortion INTRODUCED BY OPTICAL FEEDER LINK}

The effect of the non-linear distortion that the HPA (and MZM) introduces is evaluated in terms of the TD, which provides a generic way to evaluate the impact on the PER. In mathematical terms, the TD is defined as [14]

$$
\mathrm{TD}=E_{\mathrm{s}} /\left.N_{0}\right|^{\mathrm{nl}}-E_{\mathrm{s}} /\left.N_{0}\right|^{\text {awgn }}+\mathrm{OBO},
$$

where $E_{\mathrm{S}} /\left.N_{0}\right|^{\text {awgn }}$ and $E_{\mathrm{S}} /\left.N_{0}\right|^{\text {nl }}$ are the required $E_{\mathrm{S}} / N_{0}$ to achieve the target PER in case of AWGN and non-linear channel transmissions, respectively. Finally, the Output BackOff $(\mathrm{OBO})$ is the ratio between the saturation output power and the operating output power at a given IBO (of the HPA) and/or intensity modulation index $\beta$ (of the MZM).

\section{A. In-band and out-of-band non-linear distortion of MZM}

Here, we aim at characterizing the non-linear distortion that the MZM introduces for different $\beta$, including the in-band (around IF) and out-of-band distortions (odd multiples of IF). For this, we first apply the Taylor series expansion for the sine function on the Right-Hand Side (RHS) of (8) to obtain

$$
\begin{aligned}
& \sin \left(\beta s_{\mathrm{pb}}(t)\right)=\sin \left[\beta a_{\mathrm{bb}}(t) \cos \left(\omega_{\mathrm{if}} t+\varphi_{\mathrm{bb}}(t)\right)\right] \\
& \quad=\sum_{n=0}^{\infty} \frac{(-1)^{n}}{(2 n+1) !} \beta^{2 n+1} a_{\mathrm{bb}}^{2 n+1}(t) \cos ^{2 n+1}\left(\omega_{\mathrm{if}} t+\varphi_{\mathrm{bb}}(t)\right) .
\end{aligned}
$$

Then, utilizing the trigonometric identity

$$
\cos ^{2 n+1}(x)=\frac{1}{4^{n}} \sum_{k=0}^{n}\left(\begin{array}{c}
2 n+1 \\
k
\end{array}\right) \cos [(2 n+1-2 k) x],
$$

with $\left(\begin{array}{c}n \\ k\end{array}\right)=\frac{n !}{k !(n-k) !}$, it is possible to see that

$$
\begin{aligned}
\sin \left(\beta s_{\mathrm{pb}}(t)\right) & =\sum_{n=0}^{\infty} \sum_{k=0}^{n} \frac{(-1)^{n}}{(2 n-k+1) !} \frac{\beta^{2 n+1}}{k !} \frac{a_{\mathrm{bb}}^{2 n+1}(t)}{4^{n}} \\
& \times \cos \left[(2 n+1-2 k)\left(\omega_{\mathrm{if}} t+\varphi_{\mathrm{bb}}(t)\right)\right] \\
= & \sum_{n=0}^{\infty} \frac{(-1)^{n}}{(n+1) !} \frac{\beta^{2 n+1}}{n !} \frac{a_{\mathrm{bb}}^{2 n+1}(t)}{4^{n}} \cos \left[\omega_{\mathrm{if}} t+\varphi_{\mathrm{bb}}(t)\right] \\
+ & \sum_{n=1}^{\infty} \frac{(-1)^{n}}{(n+2) !} \frac{\beta^{2 n+1}(n-1) !}{(n)} \frac{a_{\mathrm{bb}}^{2 n+1}(t)}{4^{n}} \cos \left[3\left(\omega_{\mathrm{if}} t+\varphi_{\mathrm{bb}}(t)\right)\right]+\ldots \\
= & 2 J_{1}\left(\beta a_{\mathrm{bb}}(t)\right) \cos \left[\omega_{\mathrm{if}} t+\varphi_{\mathrm{bb}}(t)\right] \\
& -2 J_{3}\left(\beta a_{\mathrm{bb}}(t)\right) \cos \left[3\left(\omega_{\mathrm{if}} t+\varphi_{\mathrm{bb}}(t)\right)\right]+\ldots,
\end{aligned}
$$

where $J_{n}(x)$ is the Bessel function of the first kind with order $n$. Note that to obtain the last formula in (19), the ascending series (9.1.10) from [15] has been used, i.e.,

$$
J_{v}(z)=\left(\frac{1}{2} z\right)^{v} \sum_{k=0}^{\infty} \frac{1}{k ! \Gamma(v+k+1)}\left(-\frac{1}{4} z^{2}\right)^{k}
$$

in which $\Gamma(x)$ is the Gamma function [15]. Note that the first term in the last formula of (19) represents the in-band 
TABLE I: Parameters of the optical feeder link

\begin{tabular}{|c|l|c|c|}
\hline Symbol & Optical link parameter & Value & Unit \\
\hline$P_{\mathrm{O}, \mathrm{ld}}$ & Average optical power of LD & 46 & $\mathrm{dBm}$ \\
\hline$G_{\mathrm{O}, \mathrm{tx}}$ & Optical gain of transmitter (ground telescope) & 110 & $\mathrm{~dB}$ \\
\hline$G_{\mathrm{O}, \mathrm{rx}}$ & Optical gain of receiver (satellite telescope) & 110 & $\mathrm{~dB}$ \\
\hline$L_{\mathrm{o}, \mathrm{fso}}$ & Free space path loss of optical link & 290 & $\mathrm{~dB}$ \\
\hline$L_{\mathrm{O}, \mathrm{sys}}$ & System losses in the optical feeder link & 15 & $\mathrm{~dB}$ \\
\hline$G_{\mathrm{edfa}}$ & Gain of the optical amplifier (EDFA) & 50 & $\mathrm{~dB}$ \\
\hline$\mu$ & Responsivity of photodetector (PIN diode) & 0.5 & $\mathrm{~A} / \mathrm{W}$ \\
\hline$B_{\mathrm{e}}$ & Bandwidth of electrical filter (PIN output) & 1.5 & $\mathrm{GHz}$ \\
\hline$B_{\mathrm{O}}$ & Bandwidth of optical channel (1550 nm) & 12.5 & $\mathrm{GHz}$ \\
\hline$\rho_{\mathrm{ase}}$ & PSD of amplified spontaneous emissions & $2.0 \times 10^{-19}$ & $\mathrm{~W} / \mathrm{Hz}$ \\
\hline$\rho_{\text {rin }}$ & PSD of RIN process (normalized) & -160 & $\mathrm{~dB}$ \\
\hline$\rho_{\text {back }}$ & PSD of background noise entering EDFA & $7.6 \times 10^{-25}$ & $\mathrm{~W} / \mathrm{Hz}$ \\
\hline$i_{\mathrm{n}}$ & Electrical noise current spectral density & $1.0 \times 10^{-11}$ & $\mathrm{~A}$ \\
\hline$i_{\mathrm{dark}}$ & Dark current at the photodetector output & $1.0 \times 10^{-10}$ & $\mathrm{~A}$ \\
\hline
\end{tabular}

desired signal plus the in-band non-linear distortion (i.e., the overall signal around the IF), whereas the remaining terms identify the out-of-band non-linear distortion located around the odd multiples of the IF. Therefore, when a proper filtering is performed at the output of the HPA, we have that

$$
\begin{aligned}
& \operatorname{PBF}_{\mathrm{if}}\left\{\sin \left(\beta s_{\mathrm{pb}}(t)\right)\right\}=2 J_{1}\left(\beta a_{\mathrm{bb}}(t)\right) \cos \left[\omega_{\mathrm{if}} t+\varphi_{\mathrm{bb}}(t)\right] \\
& =\left[\beta a_{\mathrm{bb}}(t)+\sum_{k=1}^{\infty} \frac{(-1)^{k}\left(\beta a_{\mathrm{bb}}(t)\right)^{2 k+1}}{k !(k+1) ! 4^{k}}\right] \cos \left[\omega_{\mathrm{if}} t+\varphi_{\mathrm{bb}}(t)\right],(21)
\end{aligned}
$$

where $\operatorname{PBF}_{\text {if }}\{\cdot\}$ represents the passband filtering operation around the IF. Note that the sum that appears on the final formula of (21) represents the in-band (non-linear) distortion that the MZM introduces on the DVB-S2 signal.

\section{Simulation Results}

Table I summarizes the parameters of the optical feeder link, including the optical gains, attenuations, and different sources of optical noise that have been described in Section II-C. In all cases, a DPD scheme and a single-tap LMS equalizer are used in transmission and reception, respectively, to minimize the impact of the non-linear distortion on the PER of the satellite system. Without loss of generality, we focus our attention on the TD that MODCODs with 16-APSK modulation experience. The Bessel approximation presented in Section III-A is considered to model the in-band distortion that the MZM introduces on the received DVB-S2 signal. The out-of-band distortion components are neglected, as they are removed by the passband filter that is placed at the HPA output.

Figure 2 show the effect of the non-linear distortion that the MZM (HPA) introduces when the response of the HPA (MZM) is assumed ideal (i.e., perfectly linear). More precisely, Fig. 2a show the TD that the HPA introduces for different IBO when the intensity modulation index $\beta$ of the MZM is low enough to introduce negligible distortion. As expected, the weaker is the FEC encoding (e.g., 16APSK-9/10), the higher should be the IBO to minimize the TD that is experienced. It is important to highlight that these values are aligned with the ones reported in [14] for the same DVB-S2 MODCODs. On the other hand, Fig. $2 b$ show the TD that the MZM introduces for different modulation indexes $\beta$ when the IBO of the HPA is large enough to introduce negligible distortion. Based on these results, it is possible to see the the optimal modulation index $\beta_{\text {opt }}$ does not change notably for the different FEC codes that the DVB-S2 standard defines for 16-APSK modulation.

Finally, the combined effect of the non-linear distortion that both HPA and MZM introduce on the DVB-S2 system is analyzed in Fig. 3. For brevity, the performance of the most robust (i.e., 16APSK-2/3) and least robust (i.e., 16APSK-9/10) MODCODs are presented in Fig. 3a and Fig. 3b, respectively. These MODCODs represent the two extreme working conditions and, due to that, the performance of the other MODCODs that are not visualized in this paper fall between them. Since it is not easy to characterize accurately the TD as function of the IBO and $\beta$ in a 3-D plot, we present the corresponding contour curves for the corresponding DVB-S2 MODCODs. As expected, the TD that is obtained when both the MZM and HPA introduce non-linear distortion is slightly superior to the ones reported in Fig. 2a and Fig. 2b separately. However, when optimizing the working conditions of the HPA $\left(\mathrm{IBO}_{\mathrm{opt}}\right)$ and the MZM $\left(\beta_{\text {opt }}\right)$ jointly, the minimum TD that can be achieved is better than the one that is obtained when each of these parameters are optimized separately. Finally, the least robust is the FEC code that is utilized (i.e., less redundancy), the higher should be the IBO and the lower should be the intensity modulation index $\beta$ to minimize the TD of the system.

Based on the obtained simulation results, the optimal intensity modulation index $\beta$ (in linear scale) for all DVB-S2 MODCODs with 16-APSK modulation should be in the $[1.25,1.35]$ range, whereas the optimal IBO (in $\mathrm{dB}$ scale) should be in the $[2.5,4.5]$ interval. Therefore, it is possible to conclude that the main source of non-linearity in a HTS system with analog transparent (non-regenerative) optical feeder link is the HPA, and that the intensity modulation index $\beta$ does not need to take values much larger than one, to mitigate effectively the non-linear distortion in the DVB-S2 signal using DPD.

\section{CONCLUSION}

The effect of the non-linear distortion that an analog transparent (non-regenerative) optical feeder link introduces in the E2E performance of a DVB-S2 system has been studied in detail. A simple IM/DD approach was considered, where an external MZM was used to modulate the optical intensity in transmission and a PD with an EDFA was utilized in the satellite. The performance was evaluated in terms of the TD, when DPD and LMS equalization were applied to minimize the effect that the non-linear distortion has on the PER of the received DVB-S2 signal. Based on the obtained simulation results, it was possible to conclude that most of the TD in a DVB-S2 system with optical feeder link would come from the non-linear distortion that the HPA introduces (when forced to work in an energy-efficient regime - low IBO). For this case, the $\mathrm{IBO}_{\text {opt }}$ that minimized the TD of the DVB-S2 system varied notably for the different FEC codes. On the other hand, when the intensity modulation index $\beta_{\mathrm{opt}}$ was tuned to minimize the TD, the most convenient value for the different DVB-S2 MODCODs did not vary notably. Nevertheless, it is important to highlight that the selection of the optimal IBO and $\beta$ should be done jointly, in order to obtain a TD that is lower than the one that would be observed when both parameters of the MZM and HPA are optimized separately. 


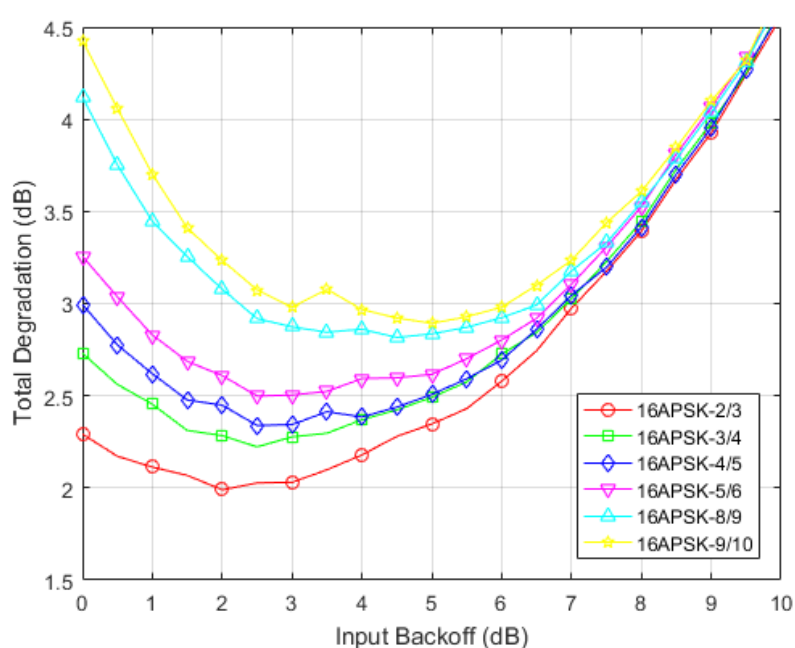

(a) Ideal optical feeder link (valid when MZM $\beta$ is low).

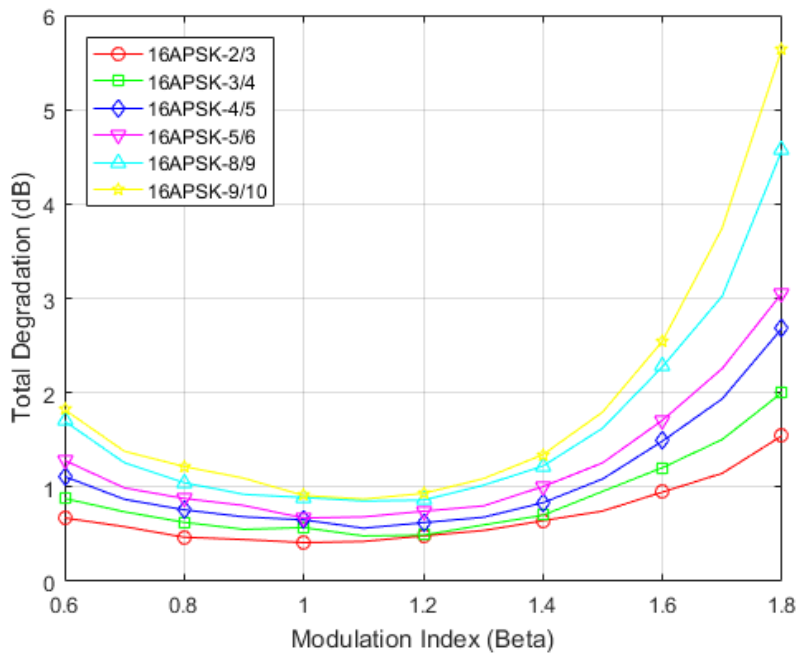

(b) Ideal RF amplification in satellite (valid when HPA IBO is large).

Fig. 2: Total degradation on the received signal assuming DPD compensation for DVB-S2 MODCODs using 16-APSK modulation.

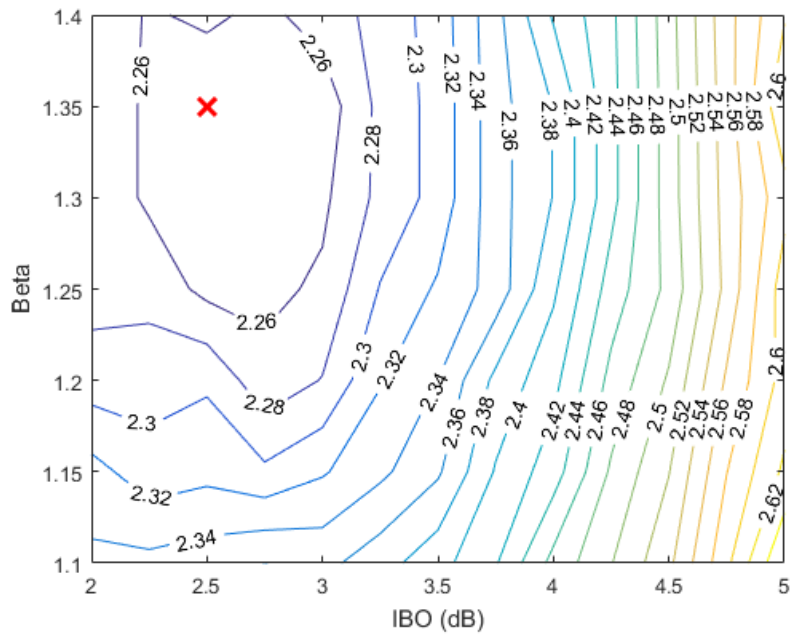

(a) $16 \mathrm{APSK}-2 / 3$ (strongest FEC). $\mathrm{TD}_{\min }=2.24 \mathrm{~dB}$ (red cross).

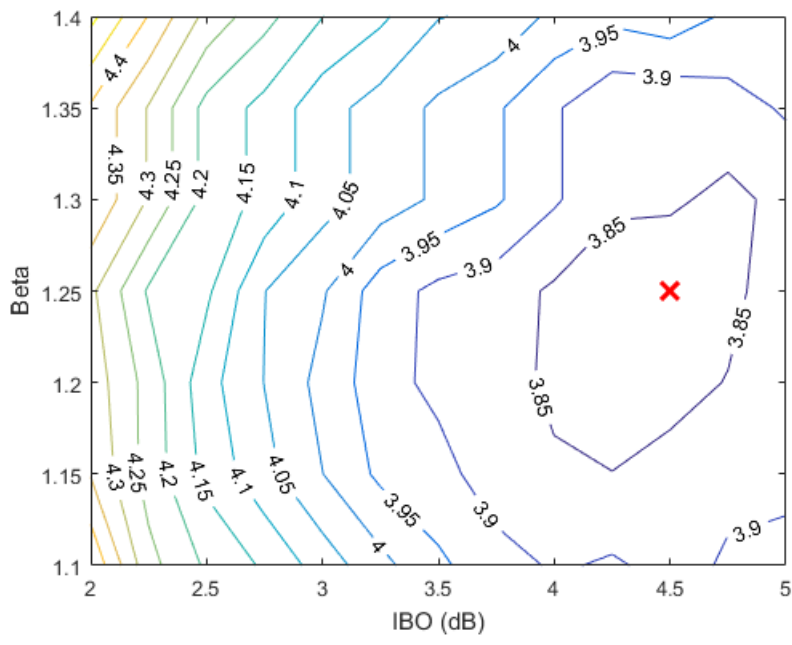

(b) 16 APSK-9/10 (weakest FEC). $\mathrm{TD}_{\min }=3.81 \mathrm{~dB}$ (red cross).

Fig. 3: Contour curves of the 3D-picture that illustrates the TD as function of the IBO (HPA) and $\beta$ (MZM) that is utilized, assuming DPD compensation and DVB-S2 MODCODS with 16-APSK modulation and different FEC codes. Red crosses show the minimum TD values.

\section{REFERENCES}

[1] M. Jia, X. Gu, Q. Guo, W. Xiang, and N. Zhang, "Broadband hybrid satellite-terrestrial communication systems based on cognitive radio toward 5G," IEEE Wireless Commun., vol. 23, no. 6, pp. 96-106, Dec. 2016.

[2] D. Giggenbach, E. Lutz, J. Poliak, R. Mata-Calvo, and C. Fuchs, "A high-throughput satellite system for serving whole Europe with fast Internet service, employing optical feeder links," in Proc. ITG Symposium on Broadband Coverage in Germany, Apr. 2015, pp. 1-7.

[3] I. Ahmad, K. Nguyen, and N. Letzepis, "Performance analysis of high throughput satellite systems with optical feeder links," in Proc. IEEE Global Commun. Conf., Dec. 2017, pp. 1-7.

[4] N. Perlot, T. Dreischer, C. Weinert, and J. Perdigues, "Optical GEO feeder link design," in Future Netw. Mobile Summit, July 2012, pp. 1-8.

[5] R. Mata-Calvo, D. Giggenbach, A. Le Pera, J. Poliak, R. Barrios, and S. Dimitrov, "Optical feeder links for very high throughput satellitesSystem perspectives," in Proc. Ka Broadband Commun., Navigation and Earth Observation Conf., Oct. 2015, pp. 1-7.

[6] S. Dimitrov, B. Matuz, G. Liva, R. Barrios, R. Mata-Calvo, and D. Giggenbach, "Digital modulation and coding for satellite optical feeder links," in Proc. Advanced Satellite Multimedia Systems Conf. and Signal Process. for Space Commun. Workshop, Sept. 2014, pp. 150-157.

[7] M. Vázquez, A. Pérez-Neira, D. Christopoulos, S. Chatzinotas, B. Ottersten, P. Arapoglou, A. Ginesi, and G. Tarocco, "Precoding in multibeam satellite communications: Present and future challenges," IEEE Wireless Commun., vol. 23, no. 6, pp. 88-95, Dec. 2016.
[8] Y. Arimoto and N. Hiromoto, "High-speed free-space laser communication," in Performance and Management of Complex Communication Networks, The International Federation for Information Processing, Ed. Boston, MA: Springer, 1998, ch. 10, pp. 175-190.

[9] A. Dowhuszko, J. Fábrega, and A. Pérez-Neira, "Integration of wireless and optical technologies to meet the requirements of $5 \mathrm{G}$ networks and beyond," in Proc. Wireless World Research Forum, Oct. 2017, pp. 1-8.

[10] R. Piazza, B. Shankar, E. Zenteno, D. Rönnow, J. Grotz, F. Zimmer, M. Grasslin, F. Heckmann, and S. Cioni, "Multicarrier digital predistortion/equalization techniques for non-linear satellite channels," in Proc. AIAA Int. Commun. Satellite System Conf., Sept. 2012, pp. 1-15.

[11] ESTI, "Digital Video Broadcasting (DVB): Second generation framing structure, channel coding and modulation system for broadcasting, interactive services, news gathering and other broadband satellite applications," Tech. Rep. EN 302207 v.1.3.1, Mar. 2013.

[12] A. Dowhuszko and A. Pérez-Neira, "Modeling the effect of non-linear distortion in a centralized RAN with analog optical fronthaul," in Proc. IEEE Global Commun. Conf., Dec. 2018, pp. 1-7.

[13] A. Mengali, "Optical feeder link satellite systems," in Link optimization in future generation satellite systems. $\mathrm{PhD}$ Thesis, University of Luxembourg, Oct. 2018, ch. 4, pp. 61-86.

[14] V. Dalakas, S. Papaharalabos, P. Mathiopoulos, E. Candreva, G. Corazza, and A. Vanelli-Coralli, "BICMC and TD comparative performance study of 16-APSK signal variants for DVB-S2 systems," IEEE Commun. Letters, vol. 19, no. 5, pp. 723-726, May 2015.

[15] M. Abramowitz and I. A. Stegun, Handbook of Mathematical Functions. National Bureau of Standards, Washington, DC, 1972. 